\title{
Segregation in Drying Binary Colloidal Droplets
}

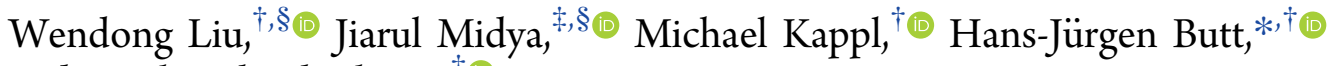 \\ and Arash Nikoubashman ${ }^{\ddagger}$
}

${ }^{\dagger}$ Department of Physics at Interfaces, Max Planck Institute for Polymer Research, Ackermannweg 10, D-55128 Mainz, Germany ${ }^{\ddagger}$ Institute of Physics, Johannes Gutenberg University Mainz, Staudingerweg 7, D-55128 Mainz, Germany

\section{Supporting Information}

\begin{abstract}
When a colloidal suspension droplet evaporates from a solid surface, it leaves a characteristic deposit in the contact region. These deposits are common and important for many applications in printing, coating, or washing. By the use of superamphiphobic surfaces as a substrate, the contact area can be reduced so that evaporation is almost radially symmetric. While drying, the droplets maintain a nearly perfect spherical shape. Here, we exploit this phenomenon to fabricate supraparticles from bidisperse colloidal aqueous suspensions. The supraparticles have a core-shell morphology. The outer region is predominantly occupied by small colloids, forming a close-packed crystalline structure. Toward the

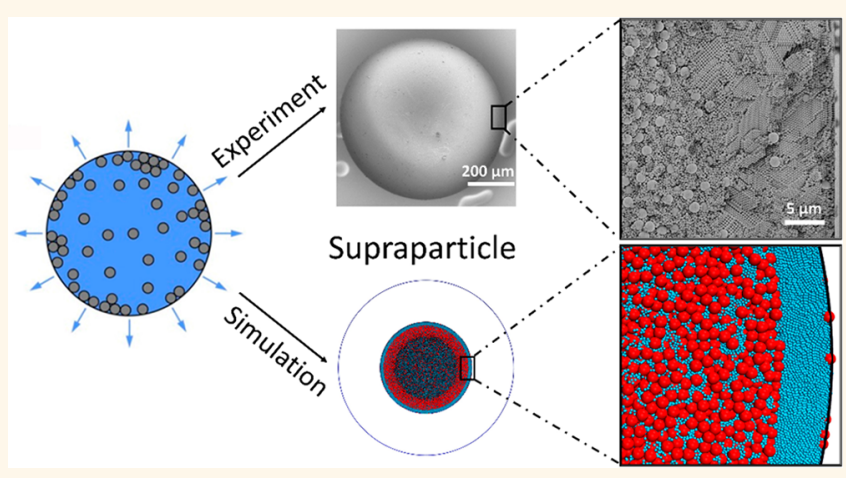
center, the number of large colloids increases and they are packed amorphously. The extent of this stratification decreases with decreasing the evaporation rate. Complementary simulations indicate that evaporation leads to a local increase in density, which, in turn, exerts stronger inward forces on the larger colloids. A comparison between experiments and simulations suggest that hydrodynamic interactions between the suspended colloids reduce the extent of stratification. Our findings are relevant for the fabrication of supraparticles for applications in the fields of chromatography, catalysis, drug delivery, photonics, and a better understanding of spray-drying.
\end{abstract}

KEYWORDS: segregation, evaporation, supraparticles, superamphiphobic, colloids

$\mathrm{W}$ hen a suspension droplet evaporates from a solid surface, the previously dispersed colloids remain on the surfaces. The structure of the deposited colloids depends on the wetting properties of the surface. If the suspension fully wets the surface, a film is formed (Figure 1a). Evaporation of films and the resulting film formation has been studied because of its relevance in applications such as painting, coating, film formation, etc. ${ }^{1}$ Due to the spatial symmetry of the drying process, evaporation can be described by a single coordinate perpendicular to the substrate. ${ }^{2-4}$

The evaporation of isolated drops occurring on partially wetted surfaces is more complex (Figure 1b). Dispersion evaporation is common in daily life and important for applications in printing, ${ }^{5}$ washing, forensics, ${ }^{6,7}$ or agriculture. ${ }^{8}$ The evaporation dynamics and the final structure of the deposit have been widely investigated both experimentally and in simulations. 9,10 For partially wetting isolated drops, the evaporation is no longer homogeneous. If the apparent contact angle of the drop is below $90^{\circ}$ more liquid evaporates at the periphery than in the center. The resulting radial flow of liquid enriches colloids at the periphery, where particles aggregate and finally form a ring- or doughnut-like structure. ${ }^{1-17}$ As a result of line pinning at the periphery, drops often evaporate at a constant contact radius rather than at a constant contact angle.

Another important case is the evaporation of free droplets, which occurs, for example, in spray drying (Figure 1d). Spray drying is a widely used process to fabricate powders from solutions or suspensions. ${ }^{18-23}$ In the case of a free spherical drop, evaporation is radially symmetric and thus can also be described by a single coordinate.

During the past decade, water and oil repellent surfaces, socalled superamphiphobic surfaces have become available. ${ }^{24,25}$ Liquid droplets on these surfaces can maintain a spherical morphology with a contact angle larger than $150^{\circ}$, while the interfacial area between liquid and solid is extremely small, i.e., $\ll V^{2 / 3}$, where $V$ is the volume of the drop (Figure 1c). Usually superamphiphobic surfaces are also porous, allowing the liquid

Received: January 17, 2019

Accepted: March 21, 2019

Published: March 21, 2019 


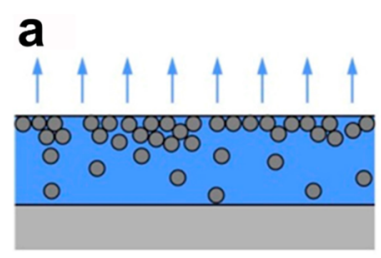

b
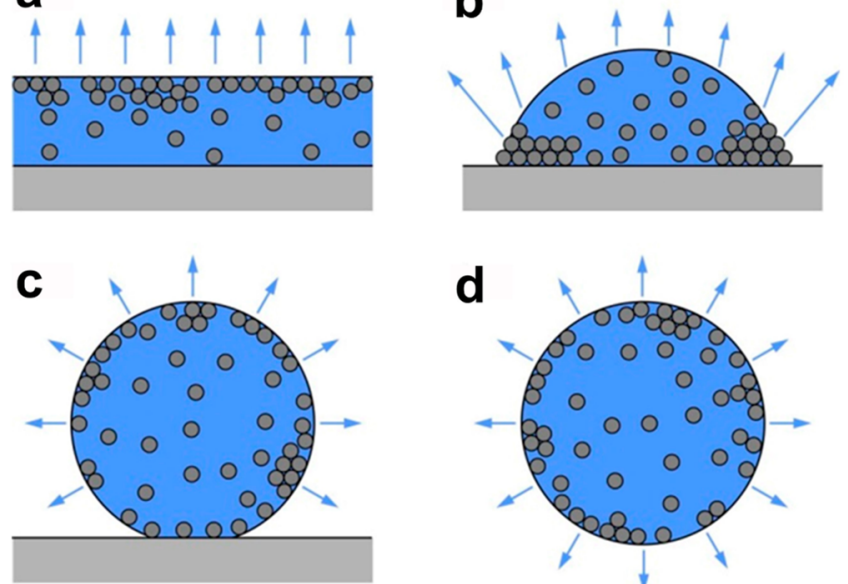

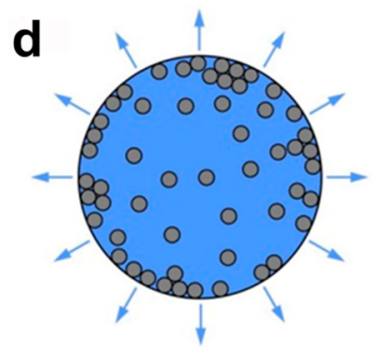

Figure 1. Schematic overview of drying suspension geometries occurring in nature and applications. Evaporation of (a) a horizontal film, (b) an isolated drop on a partially wetted surface, (c) a drop on a super liquid-repellent surface, and (d) a free drop.

to evaporate through the substrate. As a result, liquid evaporation is almost radially symmetric and the flow is suppressed. Furthermore, super-liquid-repellent surfaces allow for the fabrication of supraparticles. Such supraparticles are typically $100-1000 \mu \mathrm{m}$ in diameter and consist of colloids that are $20-2000 \mathrm{~nm}$ in size. Supraparticles were first formed from aqueous dispersions on superhydrophobic surfaces. ${ }^{26-35}$ Using superamphiphobic substrates, non-aqueous suspensions can be applied, surfactants can be added, and, due to the extremely low real contact area and the low adhesion, supraparticles can easily be removed from the surface, ${ }^{36-38}$ allowing for the production of many supraparticles. Fabrication of supraparticles on a super liquid-repellent surface has the advantage that no surrounding processing liquid is required as in the case of microfluidics. In contrast to spray drying, the process can be carried out slowly under well-controlled boundary conditions so that more-complex supraparticles can be designed. Using super-liquid-repellent surfaces for the preparation of supraparticles may also help to improve our understanding of spray drying.

Previous experimental and simulation studies about drying colloidal droplets on super-liquid-repellent surfaces have focused on monodisperse colloids. ${ }^{26,28,31,33,35}$ The final packing of the colloids depends on their interaction in suspension. For long-range repulsive interactions, the colloids tend to form a closed-packed crystalline structure. If at a certain level of concentration, attraction takes over, the colloids aggregate and form a random and more porous structure.

In this study, we aim to achieve more complex architectures of the supraparticles. We investigate both experimentally and in simulations the drying processes of a bidisperse colloidal suspension, i.e., the mixture of colloids of two distinct sizes, small (S) and large (L). Of particular interest is the distribution of colloids in the suprapaticles, i.e., whether they remain homogeneously distributed as in the initial suspension, or if they are able to form ordered structures. Previous experiments of bidisperse colloids in evaporating drops on superhydrophobic surfaces ${ }^{26}$ and in acoustically levitated drops $^{39}$ suggested an enrichment of small colloids at the supraparticle surface, but the details and mechanisms of the colloid segregation remain largely unexplored.
The evaporation of such bidisperse suspensions has been studied in more detail for thin films experimentally ${ }^{40-42}$ and in simulations. ${ }^{40,43-49}$ It was found that depending on the particle size ratios, initial volume fraction and evaporation speed, the smaller colloids end up more to the top of the film. The larger colloids are pushed to the bottom, creating "inverted" stratification. Qualitatively, this segregation occurs because evaporation leads to a local increase of the colloid concentration near the film-air interface, which, in turn, translates to a chemical potential gradient for both colloid species. ${ }^{44,45}$ Typically, this gradient is steeper for the large colloids than for the small ones because more volume is excluded to the large colloids. As a consequence, the large colloids experience a stronger driving force pushing the colloids away from the film-air interface. Inverted stratification then occurs if the mobility of the large colloids decrease slower than the driving force increases. The quantitative extent of stratification depends on the specific system, e.g., whether the dispersed particles are rigid colloids or flexible polymers ${ }^{43,45,49}$ and whether or not hydrodynamic interactions are included in the theoretical description. ${ }^{47-49}$ For a more comprehensive discussion on evaporating films, we refer to the recent review by Schulz and Keddie. ${ }^{4}$

To study the stratification in spherical drops, we investigated the evaporation behavior of drying binary colloidal drops on a superamphiphobic surface. Aqueous suspensions of polystyrene (PS) colloids of mean diameters $d_{S}=338 \mathrm{~nm}$ and $d_{\mathrm{L}}=1430 \mathrm{~nm}$ were used as a model system. Before drying, the colloidal suspension making up the droplet contained a volume fraction of $\phi_{\mathrm{S}}=2.5 \%$ for small colloids and $\phi_{\mathrm{L}}=5.5 \%$ for large colloids, respectively. At the end of the evaporation process, the total colloidal volume fraction in the dried supraparticle reached $\phi=\phi_{\mathrm{S}}+\phi_{\mathrm{L}} \approx 78 \%$. With the help of scanning electron microscopy (SEM), we observed a significant enrichment of small colloids at the supraparticle surface with a concomitant depletion of large colloids in this region. Toward the center of the supraparticles the two species gradually mixed again. The extent of this separation became less pronounced when we decreased the evaporation speed. Computer simulations confirmed this dynamic stratification and provided additional information on the colloid distribution during the evaporation process. These results will help us to prepare supraparticles for various applications such as in chromatography, catalysis, or for making photonic crystals.

\section{RESULTS AND DISCUSSION}

Evaporation of Drops. The relevant parameter describing the evaporation process of a suspension is the Péclet number (Pe). It characterizes the relative contributions of advection and diffusion to the motion of the colloids. For evaporating spherical droplets, $\mathrm{Pe}$ is the ratio of the typical time, a dispersed particle needs to diffuse one initial droplet radius, $\tau_{\mathrm{d}}$, and the characteristic time of evaporation, $\tau_{\mathrm{ev}}$. The evaporation time can be expressed as $\tau_{\mathrm{ev}}=R_{0} / v_{\mathrm{ev}}$, where $R_{0}$ is the initial droplet radius and $v_{\mathrm{ev}}$ is the speed of the receding droplet-air interface. For (free) droplets, the evaporation is limited by the diffusion of water molecules through the surrounding air, ${ }^{50}$ and the droplet volume $V$ decreases as:

$$
V^{2 / 3}=V_{0}^{2 / 3}-\alpha t
$$


where $V_{0}$ is the initial volume of the droplet and $\alpha$ is the rate of surface reduction. Taking the time derivative of eq 1 , one obtains for the initial $v_{\mathrm{ev}}$ the following expression:

$$
v_{\mathrm{ev}}=\frac{1}{2}\left(\frac{3}{4 \pi}\right)^{2 / 3} \frac{\alpha}{R_{0}}
$$

For a free, spherical droplet, $\alpha$ can be determined analytically, i.e.:

$$
\alpha_{\text {free }}=2\left(\frac{4 \pi}{3}\right)^{2 / 3} \frac{D_{\text {air }} m \Delta P}{\rho k_{\mathrm{B}} T}
$$

Here, $D_{\text {air }}$ is the diffusion coefficient of vapor molecules in air, $m$ is the mass of one vapor/liquid molecule, $\rho$ is the density of the liquid, $\Delta P$ is the difference in pressure at the droplet surface and outside the film of vapor, and $k_{\mathrm{B}} T$ is the thermal energy of the system. For sessile droplets which are shaped like a spherical cap, Picknett and Bexon ${ }^{51}$ calculated the evaporation rate and found:

$$
\alpha_{\text {sessile }}=\left(\frac{8 \pi}{3}\right)^{2 / 3} \frac{D_{\mathrm{air}} m \Delta P}{\rho k_{\mathrm{B}} T} \frac{f}{\beta^{1 / 3}}
$$

with $\beta=(1-\cos \theta)^{2}(2+\cos \theta)$ with the contact angle $\theta$, and $f=0.00008957+0.633 \theta+0.116 \theta^{2}-0.08878 \theta^{3}+$ $0.01033 \theta^{4}$ for $\theta(\mathrm{rad})$ above $10^{\circ}$. The pressure difference $\Delta P$ in eqs 3 and 4 can be expressed in terms of relative humidity $\mathrm{RH}$ as $\Delta P=(1-\mathrm{RH}) P_{0}$ with saturation vapor pressure $P_{0}$. We indeed observed a linear decrease of $V^{2 / 3}$ for the evaporation of pure water droplets as well as the colloidal suspensions (Movie S1 and Figure S1). Using eq 4 for pure water droplets at our experimental conditions $\left(\theta=154^{\circ}\right.$, $T=23{ }^{\circ} \mathrm{C}, \quad P_{0}=2810 \mathrm{~Pa}$, and $\left.\mathrm{RH}=24 \%\right)$, we find $\alpha_{\text {sessile }}=1550 \mu \mathrm{m}^{2} / \mathrm{s}$, which is in excellent agreement with the value of $\alpha=1482 \pm 7 \mu \mathrm{m}^{2} / \mathrm{s}$ that we obtained from fitting our results with eq 1 . Note that these values for $\alpha$ are roughly $30 \%$ smaller than the ones expected for completely free spherical droplets. Droplets of suspensions evaporated with the same kinetics as pure water droplets but with a slower rate of $\alpha=1046 \pm 20 \mu \mathrm{m}^{2} / \mathrm{s}$ (Figure S1d). We attribute the slight reduction in the evaporation rate to the reduced surface area of the droplets caused by colloids coating the surface.

The characteristic diffusion time can be expressed as $\tau_{\mathrm{d}}=R_{0}^{2} / D_{\mathrm{p}}$, where $D_{\mathrm{p}}$ is the diffusion coefficient of the colloids. For a spherical particle with stick boundary conditions, $D_{\mathrm{p}}$ can be estimated through the Stokes-Einstein relation, i.e., $D_{\mathrm{p}}=k_{\mathrm{B}} T /(3 \pi \eta d)$, with suspension viscosity $\eta$. For dilute suspensions of spherical particles, Cichocki and Felderhof showed that $\eta$ can be estimated within reasonable accuracy using $\eta=\eta_{\mathrm{S}}\left(1+2.5 \phi+5.913 \phi^{2}\right),{ }^{52}$ with solvent viscosity $\eta_{\mathrm{S}}$. This expression can be considered a correction of order $\phi^{2}$ to the Einstein viscosity equation. ${ }^{53,54}$ For the initial colloid volume fraction $\phi=8 \%$, we find that the suspension viscosity increases by roughly $24 \%$ compared to the neat solvent (water in our case).

In conclusion, we find $P e=\tau_{\mathrm{d}} / \tau_{\mathrm{ev}}=\frac{1}{2}\left(\frac{3}{4 \pi}\right)^{2 / 3} \frac{\alpha}{D_{\mathrm{p}}}$ for evaporating spherical droplets, which is independent of the initial droplet radius. It should be noted that the Péclet numbers quoted in this work refer to the beginning of the evaporation process when the droplets have still their initial radius, $R_{0}$, and their initial colloid volume fraction $\phi=8 \%$. As the droplet is drying, the (local) colloid volume fraction increases, resulting in a (local) increase of the suspension viscosity. Consequently, the Péclet numbers increase over time and eventually become undefined once the colloids jam or crystallize. Nevertheless, the initial Péclet numbers are useful quantities for characterizing the drying regime of the droplets.

Structure of Supraparticles. When the evaporation is completed, the final supraparticle assumes a nearly spherical shape. It took about $18 \mathrm{~min}$ for complete drying of a $2 \mu \mathrm{L}$ $\left(R_{0}=760 \mu \mathrm{m}\right)$ binary colloidal drop at ambient conditions (Figure S1d). Drops $(2 \mu \mathrm{L})$ of our dispersion led to supraparticles with a radius of $R_{\mathrm{f}}=355 \mu \mathrm{m}$ (Figure S2a). Given these processing conditions $\left(\alpha=1046 \pm 20 \mu \mathrm{m}^{2} / \mathrm{s}, D_{\mathrm{S}}\right.$ $=1.1 \times 10^{-8} \mathrm{~cm}^{2} / \mathrm{s}$ and $\left.D_{\mathrm{L}}=2.6 \times 10^{-9} \mathrm{~cm}^{2} / \mathrm{s}\right)$, we obtained $\mathrm{Pe}_{\mathrm{S}}=180$ and $\mathrm{Pe}_{\mathrm{L}}=770$ for the small and large colloids, respectively. Thus, diffusion is substantially slower than advection.

Small colloids were enriched at the surface of supraparticles (Figure 2a,b). The small colloids were assembled into close
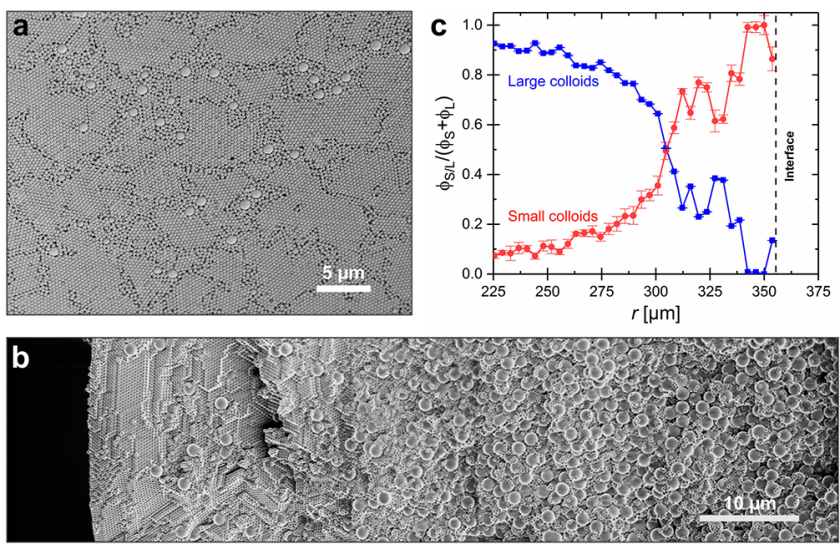

Figure 2. SEM images of a supraparticle formed from a bidisperse colloidal suspension during "fast" evaporation (24\% humidity). The diameters of the small and large colloids are $d_{\mathrm{S}}=338 \mathrm{~nm}$ and $d_{\mathrm{L}}=1430 \mathrm{~nm}$, respectively. The initial colloid volume fractions are $\phi_{\mathrm{S}}=2.5 \%$ and $\phi_{\mathrm{L}}=5.5 \%$, respectively. (a) Top view of the supraparticle surface. (b) Overlay image of crosssection from the surface to the inner part of the supraparticle, showing the stratification of small and large colloids. A moreextended cross-section showing more of the inner part is shown in Figure S3. (c) Estimated relative volume fractions of small $\left(\phi_{\mathrm{S}}\right)$ and large $\left(\phi_{\mathrm{L}}\right)$ colloids obtained from SEM images (see the Experimental Methods section for details).

packed, crystalline regions with some line and dot defects between the highly ordered regions. Only a small area of the supraparticle surface was occupied by large colloids. These seemed to anchor in particular in the defective regions between the crystals formed by the small particles.

To further analyze the distribution of small and large colloids, we cut the supraparticles in half (with a WEDO leather knife) and imaged the internal structure using SEM. Segregation of the small and large colloids was observed. The outer shell of roughly $20 \mu \mathrm{m}$ thickness contained almost exclusively small colloids (Figures $2 \mathrm{a}$ and S2b). They formed well-ordered 3D crystal structures. Only few of the large 
colloids were found in the shell. In contrast, the core part of the supraparticle consisted mostly of large colloids (Figures $2 b$, S2c, and S3). The small colloids mainly filled the interstitial spaces. No crystallization was observed in this region. Stitching of a series of SEM pictures taken along a line from the edge to the center shows the transition between these two regions in detail (Figure 2b). The radial distribution of small and large colloids in the supraparticle (Figure 2c) shows the clear spatial segregation between the two colloid types, which manifests itself during evaporation. Note that the crystallization of the smaller colloids that is observed in the outer layer of the supraparticle does not contribute to the stratification effect but is rather a consequence of the segregation. Furthermore, we did not observe any indication of buckling or cracking of the dried supraparticles in our experiments, as it can occur during spray drying due to skin formation. ${ }^{55}$ In experiments on the formation of supraparticles from monodispersed colloids, we could provoke crust formation only by pronounced acceleration of the evaporation process, using either temperatures close to the boiling point or applying vacuum (manuscript in preparation). Increasing the droplet volume to $10 \mu \mathrm{L}$ leads to a flattening of the initial droplet due to gravity. As the droplet shrinks during evaporation, it becomes spherical again as its mass decrease. The evaporation rate of $\alpha=884 \mu \mathrm{m}^{2} / \mathrm{s}$ is slightly lower than for the small droplet. However, the final deposit is nonspherical, as during the much longer evaporation time, sedimentation of the PS particles sets in (Figure S4). This sedimentation for larger drop can be mostly suppressed by density matching using $\mathrm{D}_{2} \mathrm{O}$ instead of $\mathrm{H}_{2} \mathrm{O}$, leading to almost spherical supraparticles again (Figure S5).

Comparison to Simulations. For a more-comprehensive understanding of segregation, we conducted complementary Langevin dynamics simulations with an implicit solvent. Initially, the colloids were uniformly distributed (Figure 3a and dotted line in Figure $3 \mathrm{~b}$ ). As the droplet dries, the small colloids accumulate near the surface (dashed and solid red lines in Figure $3 \mathrm{~b}$ ). A corona consisting almost exclusively of small colloids builds up. This shell is directly followed by a region enriched by large colloids (dashed and solid blue lines in Figure 3b), which extends over $\sim 15 \mu \mathrm{m}$ in the final state. Additionally, there are a few large colloids trapped at the droplet-air interface, which are no longer able to move through the dense layer of small particles. The core of the supraparticle is seemingly unaffected by the evaporation, as we find the same composition and density of colloids like in the beginning of the simulation. This behavior can be rationalized by the large evaporation rate $\left(\mathrm{Pe}_{\mathrm{S}} \gg 1\right.$ and $\left.\mathrm{Pe}_{\mathrm{L}} \gg 1\right)$, which impedes diffusive mixing of the core and shell regions.

When overlaying the density of colloids after evaporation from the simulations with the experimental measurements, the results largely agree (Figure 4). Because the Péclet number is independent of the drop radius, we scaled the radial coordinate and plotted both curves in one graph. Simulated colloid densities qualitatively agree with the experimental results. In both cases, we observe a higher density of large colloids in the core. At a distance of $\sim 80 \%$ of the supraparticle radius, the small colloids enrich and dominate the shell with the exception of a few large colloids pinned at the surface of the supraparticle.

A closer inspection of Figure 4 reveals that stratification is more pronounced in the simulations. This discrepancy likely stems from the lack of hydrodynamic interactions and liquid back flow in our simulation model. Indeed, for drying films, it
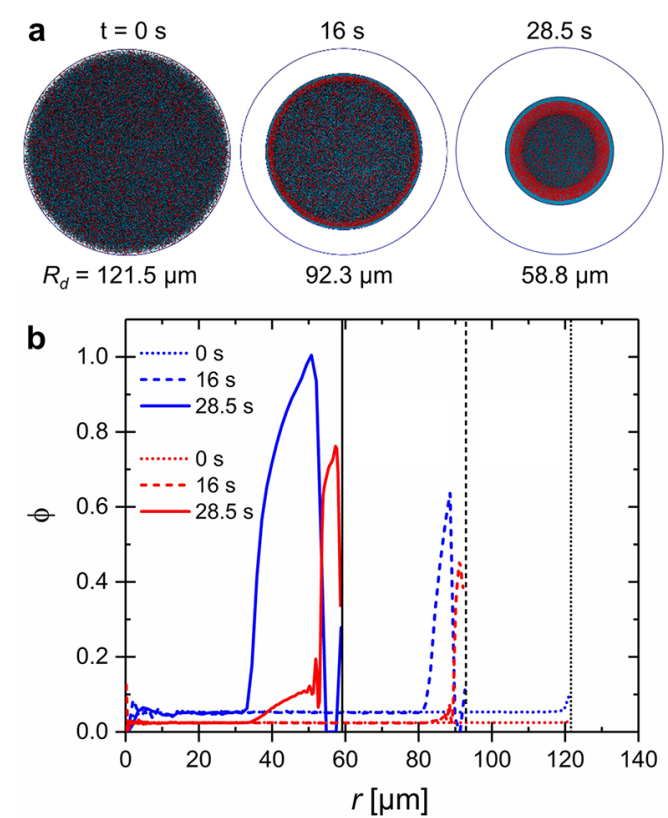

Figure 3. Simulation of a bidisperse colloidal suspension in a fast evaporation drop $\left(\alpha=1046 \mu \mathrm{m}^{2} / \mathrm{s}\right)$. The diameters of the small and large colloids are $d_{\mathrm{S}}=338 \mathrm{~nm}$ and $d_{\mathrm{L}}=1430 \mathrm{~nm}$, respectively. The initial colloid volume fractions are $\phi_{\mathrm{S}}=2.5 \%$ and $\phi_{\mathrm{L}}=5.5 \%$, respectively. (a) Cross-sections of the spherical droplet at various times during drying and the corresponding density distributions of small (cyan) and large (red) colloids. (b) Radial distribution of small (red) and large (blue) colloids at different times. The vertical black lines indicate the position of the receding droplet-air interface at the respective times.

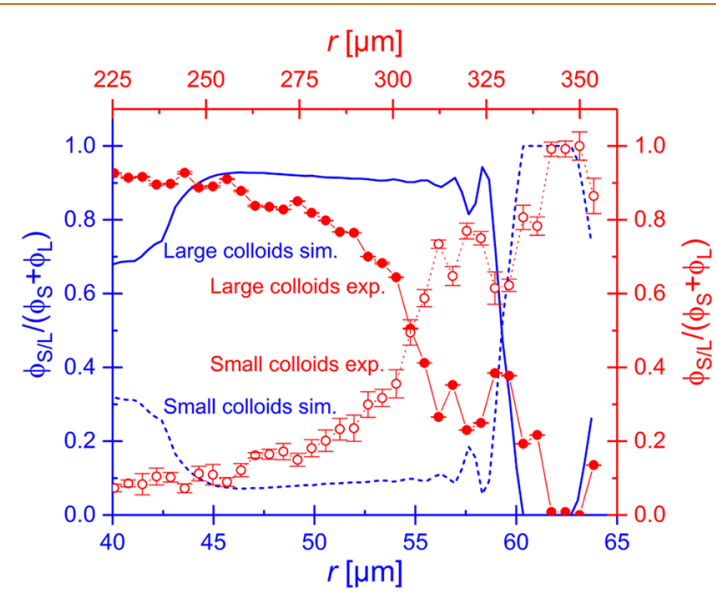

Figure 4. Relative volume fractions of colloids after evaporation from simulations (blue) and from experiments (red) versus the radial coordinate for fast evaporation $\left(\alpha=1046 \pm 20 \mu \mathrm{m}^{2} / \mathrm{s}\right)$. Solid and dashed lines correspond to data for large and small colloids, respectively.

has been shown that hydrodynamic interactions and liquid back flow can reduce the degree of stratification. ${ }^{47-49}$ The missing local hydrodynamics in the particle interaction is certainly a limitation of our model. In contrast, large-scale hydrodynamics due to Marangoni flow, as it occurs for sessile drops with pinned contact line (coffee ring effect), ${ }^{11-15}$ can be excluded in our evaporation situation with radial symmetry. Further note that the droplet radius, $R_{\mathrm{d}}$, in experiments and simulations differs by a factor of $\sim 6$ (see the Experimental 
Methods section). Although the Péclet numbers are identical in experiments and simulations, and thus qualitatively similar stratification behavior is expected, the local structuring of the dispersed colloids might be affected by the different size ratio between the colloids and the drop; each colloid takes up relatively more space in the simulations compared to the experiments, which could explain the more pronounced layering found in the simulations. The surface-to-volume ratio of the droplet is also bigger in the simulations, which further supports the stronger separation between large and small colloids in the simulations because stratification is driven by the receding boundary. Nonetheless, the agreement between experiments and simulations is rather good, underlining the robustness of the stratification process.

Low-Speed Evaporation of Binary Colloidal Droplets. For planar films, the extent of stratification depends on the evaporation speed. ${ }^{4,40,44,56}$ To observe the effect of evaporation speed in radial symmetry, we repeated our experiments at an increased relative humidity of $68 \%$. Increasing the humidity resulted in an increase in the evaporation time from $18 \mathrm{~min}$ to $2 \mathrm{~h}$ (Figure S6). The Péclet numbers for small and large colloids decreased to 27 and 110, respectively. Again, we observed a monotonic decrease of droplet volume over evaporation time, with a rate of $\alpha=154 \pm 2 \mu \mathrm{m}^{2} / \mathrm{s}$. Spherical supraparticles could still be obtained even after prolonged evaporation (Figure S7a).

For slow evaporation, the colloids were distributed more evenly in the supraparticle surface. Each large colloid was surrounded by a similar volume of small colloids (Figure 5a,b).
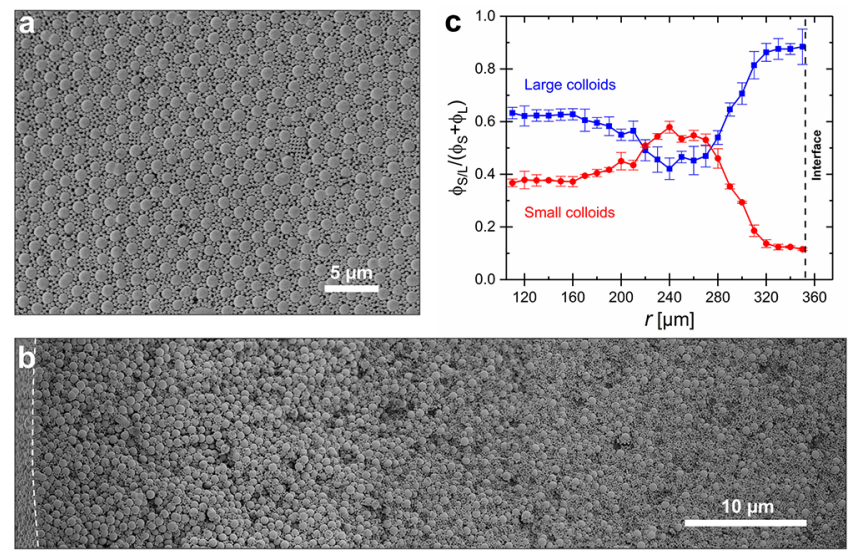

Figure 5. SEM characterization of supraparticles formed by slow evaporation (68\% humidity). The same colloid sizes and colloid volume fractions have been used as for the fast evaporation. (a) Top view of the supraparticle surface. (b) Overlay cross-view SEM images of the outer part of a supraparticle. (c) Relative volume fraction profile for large and small colloids in the supraparticle obtained under high humidity as a function of supraparticle radius.

The cross-section shows that the core region is enriched in large colloids and mixed with a low number of small colloids (Figure 5b). The difference is, however, not as pronounced as after fast evaporation. The shell between a radius of 220 and $280 \mu \mathrm{m}$ was mostly occupied by small colloids. When crossing over this layer, the number of small colloids gradually decreased, while the density of large colloids increased (Figure 5c). The small colloids did not crystallize in this case, presumably because their concentration was not high enough.
Such a weakening of the inverted stratification is consistent with simulation results (Figure S8).

\section{CONCLUSIONS}

In this study, we demonstrate that stratification of evaporating binary suspensions occurs not only for film-like geometry but also for radial symmetric geometry. An almost-radial symmetry can be achieved by evaporating drops of suspensions from superamphiphobic surfaces. The distributions of small and large colloids in the supraparticles depend on the evaporation speed. For fast evaporation, small colloids form an outer shell, while the core is enriched by large colloids. The small colloids in the shell form crystallites with several point and line defects. Only a few large colloids are trapped at the surface of the supraparticle, primarily at the site of defects. For slow evaporation, large and small colloids form a disordered structure on the surface, followed by an intermediate layer that is mostly occupied by small colloids with several large colloids randomly distributed. At the center of the supraparticles, the concentration of small colloids gradually decreases, while large colloids are enriched.

\section{EXPERIMENTAL METHODS}

Materials. Glass slides of $26 \mathrm{~mm} \times 76 \mathrm{~mm}$ in size were provided by Menzel-Gläser, Germany. Tetraethoxysilane (TEOS, reagent grade, $98 \%)$, Trichloro $(1 \mathrm{H}, 1 \mathrm{H}, 2 \mathrm{H}$, and $2 \mathrm{H}$-perfluorooctyl) silane (PFOTS, 97\%), hexadecane (reagentPlus, 99\%), and deuterium oxide $\left(\mathrm{D}_{2} \mathrm{O}, 99.9\right.$ atom \% D) were purchased from Aldrich. Ammonia $(28 \%)$, toluene, acetone, and ethanol absolute were provided by VWR CHEMICALS. N-Hexane (95\%) was purchased from Fisher Chemical. Ultrapure water was produced by Sartorius Arium 611 VF Water purification System with a resistivity of $18.2 \mathrm{M} \Omega \cdot \mathrm{cm}$. PS colloids with a diameter of $338 \mathrm{~nm}$ and $1.43 \mu \mathrm{m}$ were prepared using surfactant-free emulsion polymerization ${ }^{57}$ and purified by several cycles of centrifugation and redispersion in ultrapure water. Small and large particles therefore shared the same surface chemistry.

Preparation of Soot-Template Superamphiphobic Surfaces. Glass slides were first cleaned with toluene, acetone, and ethanol by ultrasonication, then dried under nitrogen flow. After treatment of the glass slides with oxygen plasma (Diener Electronic Femto, $300 \mathrm{~W}$ for $5 \mathrm{~min}$ ), candle soot was deposited on the glass slides. ${ }^{58}$ Afterward, the soot-coated glass slides were placed in a desiccator together with two opened glass bottles containing $3 \mathrm{~mL}$ of TEOS and aqueous ammonia solution. The desiccator was closed, evacuated to $250 \mathrm{mbar}$, and then vented with ambient air to atmospheric pressure for chemical vapor deposition of TEOS (catalyzed with ammonia) for $24 \mathrm{~h}$. After the soot was coated with a silica shell, the soot was burnt away at $550{ }^{\circ} \mathrm{C}$ for $3 \mathrm{~h}$. In this way, a fractal-like silica nanoparticle structure with overhangs was created. To achieve superamphiphobicity, the surface was further modified with trichloro $(1 \mathrm{H}, 1 \mathrm{H}, 2 \mathrm{H}$, and $2 \mathrm{H}$ perfluorooctyl) silane by means of chemical vapor deposition. Silicacoated surfaces were put into a desiccator together with an opened glass bottle containing $0.1 \mathrm{~mL}$ of the silane. The desiccator was evacuated to $50 \mathrm{mbar}$ and left for $3 \mathrm{~h}$ to finally obtain the soottemplate superamphiphobic surfaces. The superamphiphobic substrate exhibited good liquid repellent properties because it was constructed from fractal silica structure with overhangs (Figure S9a) and further modified with perfluorosilane. Water and hexadecane droplets form a spherical morphology. Water droplets possess a contact angle over $170^{\circ}$ (Figure S9b) with a sliding angle about $1^{\circ}$, while hexadecane droplets have a contact angle around $154^{\circ}$ (Figure S9c) with a sliding angle about $8^{\circ}$ at a volume of $5 \mu \mathrm{L}$.

Evaporation of Binary Colloidal Droplets on Superamphiphobic Surfaces. A binary mixture of PS colloids with a diameter of $d_{\mathrm{L}}=1.43 \mu \mathrm{m}$ ( Pdi $=0.184 \pm 0.08$, where Pdi is the polydispersity index $)$ and $d_{\mathrm{S}}=338 \mathrm{~nm}$ (Pdi $\left.=0.043 \pm 0.011\right)$ was first prepared. The volume fractions (vol \%) of large and small colloids in the water were 
$\phi_{\mathrm{L}}=5.5 \%$ and $\phi_{\mathrm{S}}=2.5 \%$, respectively. A total of $2 \mu \mathrm{L}$ of this suspension were dropped onto the superamphiphobic surface. Drops were evaporated at $23{ }^{\circ} \mathrm{C}$, either at ambient conditions (relative humidity $24 \%$ ), or at a relative humidity of $68 \%$ (measured with a TFA Digital Thermo-Hygrometer). High humidity was achieved by putting several opened water bottles around the superamphiphobic substrates in a chamber and by allowing the system to equilibrate for about $0.5 \mathrm{~h}$ before placing the drop.

Characterization. Static and sliding angles of $5 \mu \mathrm{L}$ of water and hexadecane droplets on a superamphiphobic surface were measured with a goniometer, Dataphysics OCA35 (Data Physics Instruments $\mathrm{GmbH}$, Germany). The morphology of the soot-template superamphiphobic surface and supraparticles were characterized by SEM (low-voltage LEO 1530 Gemini, Germany). The samples were sputter-coated with a $5 \mathrm{~nm}$ layer of Pt using a BalTec MED 020 coater prior to taking images to avoid charge buildup. The distributions of colloids in the supraparticles from the experiments were obtained by analyzing the SEM images using ImageJ software. The shape of the droplet during the evaporation process was recorded with a side view camera (IDS uEye USB camera). The polydispersity of the prepared PS colloids were characterized by dynamic light scattering (Malvern Zetasizer Nano S90).

Simulations. In the simulations, we modeled the colloidal suspension as a binary mixture of large and small colloids with the same size ratio $d_{\mathrm{L}} / d_{\mathrm{S}} \approx 4.2$ as in the experiments, and used $d_{\mathrm{S}}=338 \mathrm{~nm}$ as our unit of length. The colloid masses were set accordingly to $m_{\mathrm{L}} / m_{S}=\left(d_{L} / d_{S}\right)^{3}$, with $m_{\mathrm{S}}$ being the unit mass in the simulations. The colloids interacted via the purely repulsive WeeksChandler-Andersen (WCA) potential. $^{59}$ For computational efficiency, we used an implicit solvent model using Langevin dynamics simulations, which incorporates the effects of Brownian motion and viscous drag from the solvent on the colloids. The friction coefficient of the small colloid was set to $\gamma_{S}=2.5$, and it was adjusted for the large colloid according to $\gamma_{\mathrm{L}} / \gamma_{\mathrm{S}}=d_{\mathrm{L}} / d_{\mathrm{S}}$. This implicit solvent representation neglects solvent-mediated hydrodynamic interactions between the colloids. The temperature was kept constant at $T=1.0$ $\varepsilon / k_{\mathrm{B}}$, where $\varepsilon$ is the interaction strength of the WCA potential (see the Supporting Information for details). We obtained the time scale of our simulations by matching the diffusion coefficients of the small colloids at room temperature in water at infinite dilution using the Einstein relation $D_{i}=k_{\mathrm{B}} T / \gamma_{i}$, which provides $\tau \approx 0.0315 \mathrm{~s}$.

The spherical shape of the droplet in the experiments is reproduced by confining the colloids to a spherical domain with radius $R_{d}$. The air-droplet interface is modeled through a harmonic potential:

$$
U(r)= \begin{cases}\frac{k_{i}}{2}\left(r-R_{d}\right)^{2} & \text { for } r \geq R_{d} \\ 0 & \text { for } r<R_{d}\end{cases}
$$

Here, the spring constants $k_{\mathrm{i}}$ effectively control the surface tension and were chosen as $k_{\mathrm{L}} / k_{\mathrm{S}}=\left(d_{\mathrm{L}} / d_{\mathrm{S}}\right)^{2}$ with $k_{\mathrm{S}}=50 \varepsilon / d_{\mathrm{S}}^{2} \cdot{ }^{10,44,60}$ The harmonic form of eq 5 stems from geometric consideration of the change in surface area when a colloid is placed at the interface with a contact angle of $90^{\circ}$. This model neglects hydrodynamic interaction between colloids and interface but reflects repulsive interaction due to surface tension preventing a hydrophilic particle to cross the interface. Evaporation is mimicked by reducing $R_{d}$ according to eq 1 until the droplet reached its final size. Our treatment of the droplet-air interface using an external potential enforces a spherical droplet shape at all times and impedes buckling of the dried supraparticle. Note, however, that neither buckling nor cracking was observed in the experiments, which validates our simulation approach.

The initial droplet had a radius of $R_{d}=121.5 \mu \mathrm{m}$, which is roughly six times smaller than the experimental system due to computational limitations (the total number of colloids in the simulations was $N=9604239$ ). Note that this reduction in droplet size should not significantly alter the behavior of the system, as the simulations were conducted at the same Péclet number as that used in the experiments.
The initial volume fractions of the large and small colloids were chosen in line with those in the experiments, i.e., $\phi_{\mathrm{L}}=5.5 \%$ and $\phi_{\mathrm{S}}=2.5 \%$, respectively. The simulation time step was set to $\Delta t=0.005 \tau$, and all simulations were performed using the HOOMD-blue software package. ${ }^{61-63}$

\section{ASSOCIATED CONTENT}

S Supporting Information

The Supporting Information is available free of charge on the ACS Publications website at DOI: 10.1021/acsnano.9b00459.

Supplementary SEM measurements of supraparticles and superamphiphobic surface, evaporation of large volume droplets, goniometer measurement of surface wettability, evaporation speed under different humidity, and simulation of slow evaporation (PDF)

A video showing tracking of the morphology of binary colloidal droplet during water evaporation (AVI)

\section{AUTHOR INFORMATION}

\section{Corresponding Author}

*E-mail: butt@mpip-mainz.mpg.de; phone: +49-6131379111. ORCID $\odot$

Wendong Liu: 0000-0001-7297-8591

Jiarul Midya: 0000-0003-1695-9059

Michael Kappl: 0000-0001-7335-1707

Hans-Jürgen Butt: 0000-0001-5391-2618

Arash Nikoubashman: 0000-0003-0563-825X

\section{Author Contributions}

${ }^{\S}$ W.L. and J.M. contributed equally to this work. W.L., M.K., and H.-J.B. conceived and designed the experiments. W.L. performed the experiments and characterizations. J.M. and A.N. performed the simulations. W.L., J.M., M.K., A.N., and H.-J.B. cowrote the paper.

\section{Notes}

The authors declare no competing financial interest.

\section{ACKNOWLEDGMENTS}

This work was supported by the Max Planck Center for Complex Fluid Dynamics (W.L. and H.-J.B.). A.N. acknowledges funding by the German Research Foundation (DFG) under grant no. NI 1487/2-1. J.M. acknowledges funding by the Impulsfund of the Rhineland-Palatinate. We also gratefully acknowledge Gabriele Schäfer for synthesizing the colloids.

\section{REFERENCES}

(1) Jiang, S.; Van Dyk, A.; Maurice, A.; Bohling, J.; Fasano, D.; Brownell, S. Design Colloidal Particle Morphology and Self-Assembly for Coating Applications. Chem. Soc. Rev. 2017, 46, 3792-3807.

(2) Routh, A. F. Drying of Thin Colloidal Films. Rep. Prog. Phys. 2013, 76, 046603.

(3) Cardinal, C. M.; Jung, Y. D.; Ahn, K. H.; Francis, L. F. Drying Regime Maps for Particulate Coatings. AIChE J. 2010, 56, 27692780.

(4) Schulz, M.; Keddie, J. L. A Critical and Quantitative Review of the Stratification of Particles During the Drying of Colloidal Films. Soft Matter 2018, 14, 6181-6197.

(5) Singh, M.; Haverinen, H. M.; Dhagat, P.; Jabbour, G. E. Inkjet Printing-Process and Its Applications. Adv. Mater. 2010, 22, 673-685.

(6) Laan, N.; Smith, F.; Nicloux, C.; Brutin, D. Morphology of Drying Blood Pools. Forensic Sci. Int. 2016, 267, 104-109.

(7) Chen, R.; Zhang, L.; Zang, D.; Shen, W. Blood Drop Patterns: Formation and Applications. Adv. Colloid Interface Sci. 2016, 231, 114. 
(8) Wang, F.; Hu, Z.; Abarca, C.; Fefer, M.; Liu, J.; Brook, M. A.; Pelton, R. Factors Influencing Agricultural Spray Deposit Structures on Hydrophobic Surfaces. Colloids Surf., A 2018, 553, 288-294.

(9) Conway, J.; Korns, H.; Fisch, M. R. Evaporation Kinematics of Polystyrene Bead Suspensions. Langmuir 1997, 13, 426-431.

(10) Sefiane, K. Patterns From Drying Drops. Adv. Colloid Interface Sci. 2014, 206, 372-381.

(11) Li, Y.; Lv, C.; Li, Z.; Quere, D.; Zheng, Q. From Coffee Rings to Coffee Eyes. Soft Matter 2015, 11, 4669-4673.

(12) Deegan, R. D.; Bakajin, O.; Dupont, T. F.; Huber, G.; Nagel, S. R.; Witten, T. A. Capillary Flow As the Cause of Ring Stains From Dried Liquid Drops. Nature 1997, 389, 827-829.

(13) Larson, R. G. Re-Shaping the Coffee Ring. Angew. Chem., Int. Ed. 2012, 51, 2546-2548.

(14) Yunker, P. J.; Still, T.; Lohr, M. A.; Yodh, A. G. Suppression of the Coffee-Ring Effect by Shape-Dependent Capillary Interactions. Nature 2011, 476, 308-311.

(15) Zhao, M.; Yong, X. Modeling Evaporation and Particle Assembly in Colloidal Droplets. Langmuir 2017, 33, 5734-5744.

(16) Maki, K. L.; Kumar, S. Fast Evaporation of Spreading Droplets of Colloidal Suspensions. Langmuir 2011, 27, 11347-11363.

(17) Pham, T.; Kumar, S. Drying of Droplets of Colloidal Suspensions on Rough Substrates. Langmuir 2017, 33, 10061-10076. (18) Walton, D. E.; Mumford, C. J. Spray Dried ProductsCharacterization of Particle Morphology. Chem. Eng. Res. Des. 1999, 77, 21-38.

(19) Vehring, R.; Foss, W. R.; Lechuga-Ballesteros, D. Particle Formation in Spray Drying. J. Aerosol Sci. 2007, 38, 728-746.

(20) Schutyser, M. A. I.; Perdana, J.; Boom, R. M. Single Droplet Drying for Optimal Spray Drying of Enzymes and Probiotics. Trends Food Sci. Technol. 2012, 27, 73-82.

(21) Vicente, J.; Pinto, J.; Menezes, J.; Gaspar, F. Fundamental Analysis of Particle Formation in Spray Drying. Powder Technol. 2013, 247, 1-7.

(22) Biswas, P.; Sen, D.; Mazumder, S.; Basak, C. B.; Doshi, P. Temperature Mediated Morphological Transition During Drying of Spray Colloidal Droplets. Langmuir 2016, 32, 2464-2473.

(23) Lintingre, E.; Lequeux, F.; Talini, L.; Tsapis, N. Control of Particle Morphology in The Spray Drying of Colloidal Suspensions. Soft Matter 2016, 12, 7435-7444.

(24) Chu, Z.; Seeger, S. Superamphiphobic Surfaces. Chem. Soc. Rev. 2014, 43, 2784-2798.

(25) Ras, R.; Marmur, A. Non-Wettable Surfaces: Theory, Preparation, and Applications. Rsc Soft Matter Ser. 2017, 5, 1-391.

(26) Rastogi, V.; Melle, S.; Calderón, O. G.; García, A. A.; Marquez, M.; Velev, O. D. Synthesis of Light-Diffracting Assemblies from Microspheres and Nanoparticles in Droplets on a Superhydrophobic Surface. Adv. Mater. 2008, 20, 4263-4268.

(27) Rastogi, V.; Garcia, A. A.; Marquez, M.; Velev, O. D. Anisotropic Particle Synthesis Inside Droplet Templates on Superhydrophobic Surfaces. Macromol. Rapid Commun. 2010, 31, 190-195.

(28) Marin, A. G.; Gelderblom, H.; Susarrey-Arce, A.; van Houselt, A.; Lefferts, L.; Gardeniers, J. G.; Lohse, D.; Snoeijer, J. H. Building Microscopic Soccer Balls With Evaporating Colloidal Fakir Drops. Proc. Natl. Acad. Sci. U. S. A. 2012, 109, 16455-16458.

(29) Lee, D. W.; Jin, M. H.; Lee, C. B.; Oh, D.; Ryi, S. K.; Park, J. S.; Bae, J. S.; Lee, Y. J.; Park, S. J.; Choi, Y. C. Facile Synthesis of Mesoporous Silica and Titania Supraparticles by a Meniscus Templating Route on a Superhydrophobic Surface and Their Application to Adsorbents. Nanoscale 2014, 6, 3483-3487.

(30) Yu, B.; Cong, H.; Yuan, H.; Liu, X.; Peng, Q.; Zhang, X.; Xu, X.; Tian, C.; Yang, R.; Yang, S. Preparation of Doughnut-Like Nanocomposite Colloidal Crystal Particles With Enhanced Light Diffraction Using Drying Self-Assembly Method. Curr. Nanosci. 2015, $11,161-165$.

(31) Sperling, M.; Velev, O. D.; Gradzielski, M. Controlling the Shape of Evaporating Droplets by Ionic Strength: Formation of Highly Anisometric Silica Supraparticles. Angew. Chem., Int. Ed. 2014, 53, 586-590.
(32) Sperling, M.; Kim, H.-J.; Velev, O. D.; Gradzielski, M. Active Steerable Catalytic Supraparticles Shuttling on Preprogrammed Vertical Trajectories. Adv. Mater. Interfaces 2016, 3, 1600095.

(33) Sperling, M.; Papadopoulos, P.; Gradzielski, M. Understanding the Formation of Anisometric Supraparticles: A Mechanistic Look Inside Droplets Drying on a Superhydrophobic Surface. Langmuir 2016, 32, 6902-6908.

(34) Zhou, J.; Yang, J.; Gu, Z.; Zhang, G.; Wei, Y.; Yao, X.; Song, Y.; Jiang, L. Controllable Fabrication of Noniridescent Microshaped Photonic Crystal Assemblies by Dynamic Three-Phase Contact Line Behaviors on Superhydrophobic Substrates. ACS Appl. Mater. Interfaces 2015, 7, 22644-22651.

(35) Sekido, T.; Wooh, S.; Fuchs, R.; Kappl, M.; Nakamura, Y.; Butt, H. J.; Fujii, S. Controlling the Structure of Supraballs by $\mathrm{pH}$ Responsive Particle Assembly. Langmuir 2017, 33, 1995-2002.

(36) Deng, X.; Paven, M.; Papadopoulos, P.; Ye, M.; Wu, S.; Schuster, T.; Klapper, M.; Vollmer, D.; Butt, H. J. Solvent-Free Synthesis of Microparticles on Superamphiphobic Surfaces. Angew. Chem., Int. Ed. 2013, 52, 11286-11289.

(37) Costa, A. M.; Mano, J. F. Solvent-Free Strategy Yields Size and Shape-Uniform Capsules. J. Am. Chem. Soc. 2017, 139, 1057-1060.

(38) Wooh, S.; Huesmann, H.; Tahir, M. N.; Paven, M.; Wichmann, K.; Vollmer, D.; Tremel, W.; Papadopoulos, P.; Butt, H. J. Synthesis of Mesoporous Supraparticles on Superamphiphobic Surfaces. Adv. Mater. 2015, 27, 7338-7343.

(39) Raju, L. T.; Chakraborty, S.; Pathak, B.; Basu, S. Controlling Self-Assembly and Topology at Micro-Nano Length Scales Using a Contact-Free Mixed Nanocolloid Droplet Architecture. Langmuir 2018, 34, 5323-5333.

(40) Fortini, A.; Martin-Fabiani, I.; De La Haye, J. L.; Dugas, P. Y.; Lansalot, M.; D’Agosto, F.; Bourgeat-Lami, E.; Keddie, J. L.; Sear, R. P. Dynamic Stratification in Drying Films of Colloidal Mixtures. Phys. Rev. Lett. 2016, 116, 118301.

(41) Liu, X.; Liu, W.; Carr, A. J.; Santiago Vazquez, D.; Nykypanchuk, D.; Majewski, P. W.; Routh, A. F.; Bhatia, S. R. Stratification During Evaporative Assembly of Multicomponent Nanoparticle Films. J. Colloid Interface Sci. 2018, 515, 70-77.

(42) Carr, A. J.; Liu, W.; Yager, K. G.; Routh, A. F.; Bhatia, S. R. Evidence of Stratification in Binary Colloidal Films from Microbeam X-ray Scattering: Toward Optimizing the Evaporative Assembly Processes for Coatings. ACS Appl. Nano Mater. 2018, 1, 4211-4217.

(43) Cheng, S.; Grest, G. S. Dispersing Nanoparticles in a Polymer Film via Solvent Evaporation. ACS Macro Lett. 2016, 5, 694-698.

(44) Howard, M. P.; Nikoubashman, A.; Panagiotopoulos, A. Z. Stratification Dynamics in Drying Colloidal Mixtures. Langmuir 2017, 33, 3685-3693.

(45) Howard, M. P.; Nikoubashman, A.; Panagiotopoulos, A. Z. Stratification in Drying Polymer-Polymer and Colloid-Polymer Mixtures. Langmuir 2017, 33, 11390-11398.

(46) Tatsumi, R.; Iwao, T.; Koike, O.; Yamaguchi, Y.; Tsuji, Y. Effects of the Evaporation Rate on the Segregation in Drying Bimodal Colloidal Suspensions. Appl. Phys. Lett. 2018, 112, 053702.

(47) Sear, R. P.; Warren, P. B. Diffusiophoresis in Nonadsorbing Polymer Solutions: The Asakura-Oosawa Model and Stratification in Drying Films. Phys. Rev. E: Stat. Phys., Plasmas, Fluids, Relat. Interdiscip. Top. 2017, 96, 062602.

(48) Tang, Y.; Grest, G. S.; Cheng, S. Stratification in Drying Films Containing Bidisperse Mixtures of Nanoparticles. Langmuir 2018, 34, 7161-7170.

(49) Statt, A.; Howard, M. P.; Panagiotopoulos, A. Z. Influence of Hydrodynamic Interactions on Stratification in Drying Mixtures. J. Chem. Phys. 2018, 149, 024902.

(50) Langmuir, I. The Evaporation of Small Spheres. Phys. Rev. 1918, 12, 368-370.

(51) Picknett, R. G.; Bexon, R. The Evaporation of Sessile or Pendant Drops in Still Air. J. Colloid Interface Sci. 1977, 61, 336-350.

(52) Cichocki, B.; Felderhof, B. U. Long-Time Self-Diffusion Coefficient and Zero-Frequency Viscosity of Dilute Suspensions of Spherical Brownian Particles. J. Chem. Phys. 1988, 89, 3705-3709. 
(53) Einstein, A. Eine neue Bestimmung der Moleküldimensionen. Ann. Phys. (Berlin, Ger.) 1906, 324, 289-306.

(54) Einstein, A. Berichtigung zu meiner Arbeit: "Eine neue Bestimmung der Moleküldimensionen”. Ann. Phys. (Berlin, Ger.) 1911, 339, 591-592.

(55) Lanotte, L.; Boissel, F.; Schuck, P.; Jeantet, R.; Le FlochFouéré, C. Drying-Induced Mechanisms of Skin Formation in Mixtures of High Protein Dairy Powders. Colloids Surf., A 2018, $553,20-27$.

(56) Zhou, J.; Jiang, Y.; Doi, M. Cross Interaction Drives Stratification in Drying Film of Binary Colloidal Mixtures. Phys. Rev. Lett. 2017, 118, 108002.

(57) Shouldice, G. T. D.; Vandezande, G. A.; Rudin, A. Practical Aspects of the Emulsifier-Free Emulsion Polymerization of Stryene. Eur. Polym. J. 1994, 30, 179-183.

(58) Deng, X.; Mammen, L.; Butt, H.-J.; Vollmer, D. Candle Soot as a Template for a Transparent Robust Superamphiphobic Coating. Science 2012, 335, 67-70.

(59) Weeks, J. D.; Chandler, D.; Andersen, H. C. Role of Repulsive Forces in Determining the Equilibrium Structure of Simple Liquids. J. Chem. Phys. 1971, 54, 5237-5247.

(60) Pieranski, P. Two-Dimensional Interfacial Colloidal Crystals. Phys. Rev. Lett. 1980, 45, 569-572.

(61) Anderson, J. A.; Lorenz, C. D.; Travesset, A. General Purpose Molecular Dynamics Simulations Fully Implemented on Graphics Processing Units. J. Comput. Phys. 2008, 227, 5342-5359.

(62) Glaser, J.; Nguyen, T. D.; Anderson, J. A.; Lui, P.; Spiga, F.; Millan, J. A.; Morse, D. C.; Glotzer, S. C. Strong Scaling of GeneralPurpose Molecular Dynamics Simulations on GPUs. Comput. Phys. Commun. 2015, 192, 97-107.

(63) Howard, M. P.; Anderson, J. A.; Nikoubashman, A.; Glotzer, S. C.; Panagiotopoulos, A. Z. Efficient Neighbor List Calculation for Molecular Simulation of Colloidal Systems Using Graphics Processing Units. Comput. Phys. Commun. 2016, 203, 45-52. 